\title{
A Fourier series model for forecasting solid waste generation in the Kumasi metropolis of Ghana
}

\author{
D. Asante-Darko ${ }^{1}$, E. S. Adabor ${ }^{1} \&$ S. K. Amponsah ${ }^{2}$ \\ ${ }^{1}$ Ghana Institute of Management and Public Administration, Ghana \\ ${ }^{2}$ Kwame Nkrumah University of Science and Technology, Ghana
}

\begin{abstract}
Successful planning of a solid waste management system depends on the accuracy of prediction of solid waste generation. With a continual economic development and increase in the living standards, the demand for goods and services is increasing at an unprecedented rate, resulting in a commensurate increase in per capita waste generation. In order to facilitate informed decision making for effective solid waste management, we propose a Fourier series model to forecast solid waste generation in Kumasi, Ghana. A monthly solid waste data from 2007 to 2014 was obtained from the solid waste department of the Kumasi Metropolitan Assembly, Ghana. This was used to formulate the Fourier series model for forecasting solid waste. This approach incorporates the characteristics of the data making them more appropriate for forecasting solid waste. It was found that out of the 84 periods considered in the Fourier series model, period 42 was the best model for forecasting solid waste generation. The 1 year monthly forecast revealed that the generation of solid waste will increase as a result of the high rate of urbanization and population growth.
\end{abstract}

Keywords: solid waste, Fourier series, waste management, forecasting.

\section{Introduction}

Solid waste management is a global challenge and the situation is worse in urban areas of the developing countries where, in most cases, there are no data of how much solid waste is generated over a specific period of time. This calls for a means 
for anticipating the solid waste to be generated in order for the authorities to take proactive actions in managing the solid waste [16].

Successful planning of a solid waste management system depends critically on the prediction accuracy of solid waste generation. Prediction of municipal solid waste generation provides the basic data on which waste management system is planned, designed and operated thus, the waste transportation trucks required, segregation plant capacity, land requirements for compositing, capacity of landfill site are directly depend on the quantity of municipal waste [17].

Planning and design of municipal solid waste management systems require accurate prediction of solid waste generation [3]. Yet achieving the anticipated prediction accuracy with regard to the generation trends facing many fast-growing regions is quite challenging. The lack of complete historical records of solid waste quantity and quality due to insufficient budget and unavailable management capacity has, in his submission, resulted in a situation that makes the long-term system planning and/or short-term expansion programs intangible.

Thus, [2] proposed that to effectively handle these problems based on limited data samples, a new analytical approach capable of addressing socio-economic and environmental situations must be developed and applied for fulfilling the prediction analysis of solid waste generation with reasonable accuracy.

The prediction condition of generation trend in many developing countries (such as Ghana) is different from those in developed countries. The lack of sampling and analysis of waste in many developing countries due to insufficient budget and the lack of requisite management task force has resulted in a situation where the historical record of solid waste generation and composition can never be completed in the long term. This present a world of challenges when dealing with the subject of waste management let alone the issue of waste prediction, especially in the developing countries [4].

Waste management is a complex process that requires a lot of information from various sources such as factors on waste generation and waste quantity forecasts. When operations related to promotion of waste management systems are considered, it is observed that generation of waste and planning are found to be influenced by different factors including socio demographics [2]. These have led to numerous studies which sough to predict the quantity of solid waste generation worldwide, with the time series and the Fuzzy logic approach being the most common.

For instance, [1] used ARMA/ARIMA and exponential smoothing models to forecast solid waste generation in Arusha city Tanzania. The past data used were monthly amounts of solid waste collected by the city authorities from year 2008 to 2013. The results obtained from the extensive study indicated that ARIMA $(1,1,1)$ outperformed other potential models in terms of MAPE, MAD and RMSE measures and hence used to forecast the amount of the solid waste generation for the next years. [5] also used ARIMA time series model to explore the dynamics of solid waste generation in the Kumasi metropolitan assembly with a monthly solid waste data from 2005 to 2010. 
Other studies that have applied the Time series (ARIMA) models include [12] who used the Time Series ARIMA model approach to forecast municipal solid waste generation. The works by $[1,12]$ suggested that ARIMA $(1,1,1)$ model is the best model among all parametric time series models for forecasting solid waste generation though they assumed that the solid waste generation data follows a stationary stochastic process. That is, its mean and variance do not change over time.

However, the solid wasted generation data has a non-parametric characteristics. This implies that they are seasonal or time heterogeneous. For this reason, other researchers including $[4,10,11]$ used the fuzzy logic to predict the expected quantity of solid waste.

In a quest to get a model that best predicts solid waste generation, some researches have explored the applicability of other models including the Artificial neural networks $[13,16]$, linear programming model $[6,13-15]$ and the waste management system [7].

Clearly, there is no convergence in literature in terms of the method that can be best applied in the prediction of solid waste. This paper proposes forecasting solid waste generation will require that model capture the underlying characteristics of data. This includes the non-parametric characteristics, the time heterogeneous nature and the periodicity of data. Our approach, the Fourier series model accounts for these characteristics in the data set. This paper, firstly, develops a model that can best describe the quantity of solid waste generated using monthly data in the Kumasi metropolis. Secondly we evaluate the proposed model by comparing its sum of squared error (SSE) with the SSE of high performing existing approach namely the ARIMA time series. Finally, we apply our model to estimate the expected quantity of solid waste generation in the Kumasi metropolis for the year 2016.

\section{Method}

\subsection{The Fourier series model}

Fourier series is named in honor of Joseph Fourier, who made important contributions to the study of trigonometric series, after preliminary investigations by Leonhard Euler, Jean le Rond d'Alembert and Daniel Bernoulli. Fourier applied this technique to find the solution of the heat equation [15].

The founding principle behind the field of Fourier series analysis, is an infinite expansion of a periodic function in terms of sines and cosines or imaginary exponentials. It also makes use of the orthogonality relationships of the sines and the cosines.

James Walker [8] presented the Fourier series of a function as represented Equation (1).

$$
f(x)=a_{0}+\sum_{k=1}^{K}\left(a_{k} \cos \frac{2 \pi k}{n} x+b_{k} \sin \frac{2 \pi k}{n} x\right)
$$


where $f(x)$ is a periodic function,

$$
\begin{aligned}
& a_{n}=\frac{1}{\pi} \int_{-\pi}^{\pi} f(x) \cos n x d x, \quad n=1,2,3, \ldots, \\
& b_{n}=\frac{1}{\pi} \int_{-\pi}^{\pi} f(x) \sin n x d x, \quad n=1,2,3, \ldots .
\end{aligned}
$$

For the purpose of this research the discrete decomposition of Fourier series is considered since we dealing with the monthly tonnage solid waste generated from January 2007 to December 2014. The Fourier series for continuous function is then decomposed to give us a discrete representation which is given by Equation (4).

$$
Q_{s}^{d, s}=a_{0}+\sum_{k=1}^{K}\left(a_{k} \cos \frac{2 \pi k}{n} m+b_{k} \sin \frac{2 \pi k}{n} m\right)
$$

where

$a_{0}$ is the mean value of the seasonal cycle,

$m=1,2,3, \ldots, 84$,

$n=$ the total number of periods,

$Q_{s}^{d, s}=$ the discrete values to forecast,

$K=$ the harmonic being considered,

$$
\begin{aligned}
a_{k} & =\frac{2}{n} \sum_{m=1}^{n}\left(Q_{m}^{d, o b s} \cos \frac{2 \pi k}{n} m\right), \\
b_{k} & =\frac{2}{n} \sum_{m=1}^{n}\left(Q_{m}^{d, o b s} \sin \frac{2 \pi k}{n} m\right),
\end{aligned}
$$

$Q_{m}^{d, o b s}=$ the discrete values observed,

$m=$ the individual data points,

$n=$ the total observed data points,

$k=$ the period being considered.

As each new harmonic (period) was added to the Fourier series, the sum of square errors were determined. The sum of square errors will be used in identifying the optimal period $(k)$ associated with the Fourier series function. This is achieved by measuring the deviation between the prediction of the Fourier series function at each period $(k)$ and the observed prediction.

$$
S S E=\sum_{k=1}^{\infty}\left(Y-Y^{*}\right)^{2}
$$

where $Y$ is the observed value and $Y^{*}$ is the predicted value. This leads to the formulation in Equation (8) used in the research.

$$
S S E=\sum_{k=1}^{\infty}\left(Q_{s}^{d, o b s}-Q_{s}^{f, s}\right)^{2}
$$


where

$Q_{s}^{d, o b s}=$ observed solid waste generated,

$Q_{s}^{f, s}=$ predicted solid waste generation.

The smaller the SSE, the better the predictive ability of the Fourier series function. In the concept of series, the Fourier coefficients are unique. This Uniqueness is what differentiates one series from another (Weierstrass Approximation Theorem). In other words, what will make this Fourier series model unique to Kumasi is the unique Fourier coefficients. This means to apply Fourier series to any other location the Fourier coefficients have to be determined.

The number of periods $(k)$ used is actually determined by the number of individual data points considered. Since we considered 84 data points for this study, our period $(k)$ must run from 1 to 84 . The essence hear is to be able to get a Fourier series model that is able to estimate all the individual data points. This model will then be referred to as a good representation and then used for forecasting based on the fact that we have 84 data points. It means we have 84 models and 84 different graphical interpretation that goes to inform the strength of the model. We present the best 4 out of the 84 possible models have been presented namely $6,12,42$ and 84 . The other models are presented as supplementary files.

\section{Results and discussion}

\subsection{Study area and background of data}

Kumasi is the capital city of the Ashanti region. Tradition is held very high in Kumasi and blends very well with modernity. There is a wide range of attractions in Kumasi. Kumasi is located in the transitional forest zone and is about $270 \mathrm{~km}$ north of the national capital, Accra. It is between latitude $6.35^{\circ}-6.40^{\circ}$ and longitude $1.30^{\circ}-1.35^{\circ}$, an elevation which ranges between 250 and 300 meters above sea level with an area of about $254 \mathrm{~km}^{2}$. Kumasi features a tropical wet and dry climate, with relatively constant temperatures throughout the course of the year. Kumasi averages around $1400 \mathrm{~mm}$ (55 inches) of rain per year. The city is a rapidly growing one with an annual growth rate of $2.7 \%$ and a $3.5 \%$ rate of urbanization.

The population of Kumasi is about 4,780,380 as of 2010. In the year 2005, 1,000 tons of solid waste was generated each day in the city; three years later, it increased by $20 \%$ resulting in 1,200 tons a day. As at 2014, 4,000 tons of solid waste was being generated daily in the Kumasi Metropolis [9]. According to [5], KMA is not able to manage solid waste effectively due to rapid urbanization, poor financing capacity of authorities and lack of safe waste disposal sites, which is a reflection of the weak solid waste generation data of the city.

The lack of proper waste data eventually leads to the inability of the authorities to accurately forecast the quantity of waste to be generated. The cyclical mantra of planning is thus invoked: planning to predict or predicting to plan. 


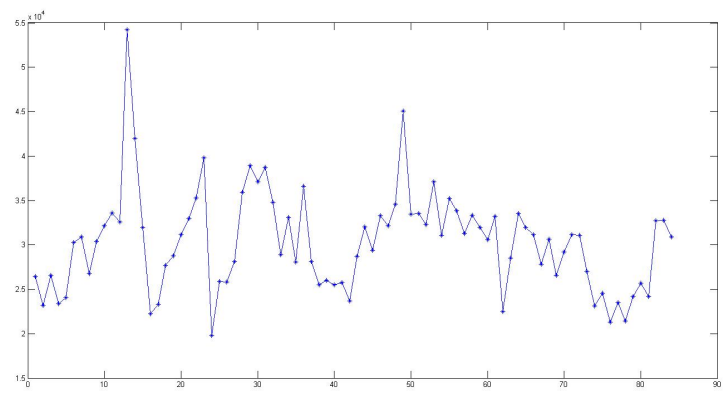

Figure 1: Time plot for solid waste for 84 months.

\subsection{Model formulation}

A time plot of the monthly solid waste generated in the Kumasi metropolis for the 84 months is presented in Figure 1. It can be observed from the time plot in Figure 1 that the data is periodic with its highest value being 54,203 tons occurring in January 2009. This peak value can be associated to the Christmas and new year festivities which comes along with high rate of consumptions of goods. A lowest value of 19,807 tons occurred in December of the same year.

We, firstly, model a Fourier series function that gives a very good representation of the time plot (Figure 1). This will be a model from Fourier series that best describes the trend of solid waste generation in the Kumasi municipality. This model obtained will then be used to predict the quantity of solid waste to be generated in the next two (2) years.

Fourier series in its definition is simply a sinusoidal representation of a periodic function,this optimal representation of a periodic function is found by alternating the value of $\mathrm{k}$ in the Fourier Series. The least sum of squared error is used to ensure a suitable $k$ is chosen. For instance at $k=1$, one will obtain a predictive Fourier series function made up of $a_{o}$ and just two other Fourier coefficients that is $a_{1}$ and $b_{1}$.

$$
f(x)=a_{0}+a_{1} \cos \frac{2 \pi}{n} m+b_{1} \sin \frac{2 \pi}{n} m
$$

When the period is increased to $k=2$, we will realize a change in the Fourier function since this will yield an increase in the number of Fourier coefficients, thus $a_{0}, a_{1}, a_{2}, b_{1}$ and $b_{2}$. Hence, Equation (10):

$$
f(x)=a_{0}+a_{1} \cos \frac{2 \pi}{n} m+a_{2} \cos \frac{2 \pi}{n} m+b_{1} \sin \frac{2 \pi}{n} m+b_{2} \sin \frac{2 \pi}{n} m
$$

The periods are progressively increased for every single period point under consideration from 1 through to 84 but selected periods with their corresponding time plots, thus periods 6,12 , and 42 are displayed, as a sample representation of all 84 computations, to show the gradual progression to the ideal curve. 


\subsubsection{Modeling Fourier series at $\boldsymbol{k}=\mathbf{6}$}

At period $6, k=6$, six (6) Fourier coefficients were obtained.These coefficients was used to build a predictive Fourier series function, the Fourier function obtained using $k=6$ was used to forecast the expected waste and the least sum of square errors was also computed to enable choose the optimum point out of the 84 periods being considered.

$$
\begin{aligned}
Q_{m}^{f, s}= & 3.027 * 10^{4}+\left(-2.1058 * 10^{3}\right) \cos \frac{2 \pi}{84} m+\left(-1.5608 * 10^{3}\right) \cos \frac{2 \pi}{84} m \\
& +\left(0.4705 * 10^{3}\right) \cos \frac{2 \pi}{84} m+\left(-2.2247 * 10^{3}\right) \cos \frac{2 \pi}{84} m \\
& +\left(1.7951 * 10^{3}\right) \cos \frac{2 \pi}{84} m+\left(0.8545 * 10^{3}\right) \cos \frac{2 \pi}{84} m \\
& +\left(0.7631 * 10^{3}\right) \sin \frac{2 \pi}{84} m+\left(2.1033 * 10^{3}\right) \sin \frac{2 \pi}{84} m \\
& +\left(0.3757 * 10^{3}\right) \sin \frac{2 \pi}{84} m+\left(0.5193 * 10^{3}\right) \sin \frac{2 \pi}{84} m \\
& +\left(-1.7134 * 10^{3}\right) \sin \frac{2 \pi}{84} m+\left(-0.3859 * 10^{3}\right) \sin \frac{2 \pi}{84} m
\end{aligned}
$$

It is clear from the observation of Figure 2, that the difference between the Fourier series prediction denoted by the color red and the actual time plot of Figure 1 is huge at period $6(k=6)$, this huge variance is further established statistically with the least squared method where we obtained a value of $2.8968 *$ $10^{7}$. This Fourier series prediction at $(k=6)$ was rejected since the deviation between the Fourier series prediction and observed values were huge, characterize by a large SSE. Hence, we increased our period $(k)$ from 6 to 12 .

\subsubsection{Modeling Fourier series at $k=12$}

At period $(k=12)$ Fourier series coefficients were obtained in addition to $a_{0}$ to construct the Fourier series predictive function as shown in Table 2. This function was used to obtained a predictive graph illustrated by Figure 3 .

At point $k=12$.

It is observed from Figure 3 that the Fourier series prediction of period 12 $(k=12)$ performed better than that of Figure 1 of $(k=6)$. The SSE between the Fourier series prediction denoted by the color red and the actual time plot of Figure 1 is not as huge as that of Figure 2. This fact was further substantiated statistically when the least sum of square error of Figure 3 was found to be $2.6746 * 10^{7}$, which is less than that of Figure 2. This Fourier Series prediction at $(k=12)$ was rejected since its deviation was not the smallest in all the 84 periods considered. Hence, it is increased from 12 to 42 .

\subsubsection{Modeling Fourier series at $k=42$}

An optimal point was finally obtained at period $(k=42)$. This period gave the smallest deviation in all the 84 periods considered,with a numerical value of 


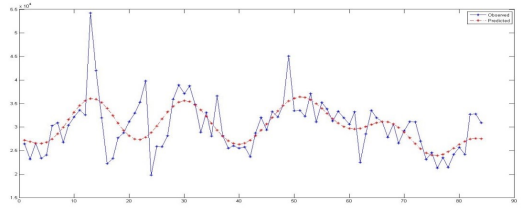

Figure 2: Fourier function prediction for period 6 .

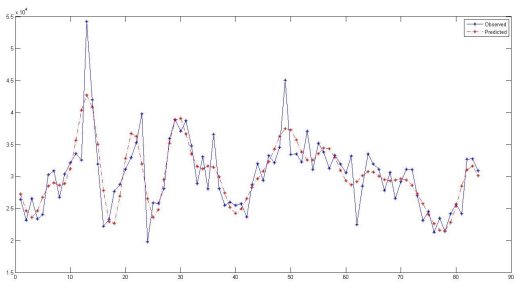

Figure 3: Fourier Function prediction for period 12.

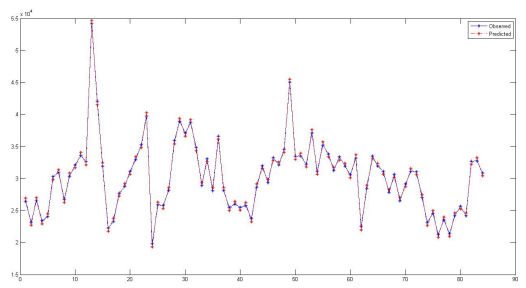

Figure 4: Fourier function prediction for period 42 .

$1.8124 * 10^{7}$. It can be observed that the Fourier series function obtained for this period gave a perfect prediction of Figure 4 . The difference between the Fourier series prediction and the time plot of Figure 1 is almost negligible. The 42 Fourier coefficients obtained for this Fourier series predictive function was then used for the construction of a 2 year monthly Predictive Fourier series model.

Table 1 below present the SSE associated with each period $(k)$ considered. Period 42 gave the smallest SSE among the SSE presented.

Table 1: A tabular representation of the sum of squared errors for model selection.

\begin{tabular}{cc}
\hline $\begin{array}{c}\text { Various iterations } \\
\text { (at various periodic values) }\end{array}$ & $\begin{array}{c}\text { Sum of } \\
\text { squared error }\end{array}$ \\
\hline 6 & $2.8968 * 10^{7}$ \\
12 & $2.6746 * 10^{7}$ \\
42 & $1.8124 * 10^{7}$ \\
\hline
\end{tabular}




\subsubsection{The Fourier Series model for forecasting solid waste generation}

In order to forecast the solid waste, an extrapolation is carried out with respect to $\mathrm{n}$ being the interval. Since our data points end at 84 , the forecast then starts from the 85th month which represents the month of January in the year 2015.

The model for forecasting is then represented by:

$$
Q_{m}^{f, s}=a_{0}+\sum_{k=1}^{42}\left(a_{k} \cos \frac{2 \pi k}{n} m+a_{k} \sin \frac{2 \pi k}{n} m\right)
$$

where $a_{k}$ and $b_{k}$ are the coefficients of the model, $n=84$ data points.

\subsection{Evaluation of model}

In this section the applicability, suitability and performance of the Fourier series model is verified by comparing it to a known high performing existing model the ARIMA Time series model. This ARIMA Time series has been widely used in research in forecasting including the area of solid waste generation [8]. The high performance of ARIMA time series models make them useful for evaluating the proposed model.

\subsubsection{Performance indicators}

The performance of the models are indicated by the SSE, the smaller the SSE the better the model. In this section, we build an Arima time series model using the same data set of solid waste generation from January 2007 to December 2014. The SSE from the ARIMA time series model and the Fourier Series Model are compared the model with smallest SSE is the best model for forecasting solid waste generation. That is the SSE gives a measure of the suitability of the model for forecasting solid waste generation. The SSE associated with each model is presented in Table 1.

\subsubsection{ARIMA time series model}

In reference to the time plot of Figure 1, an ARIMA model is identified. The model identification process is where the structure and order of the possible models are mainly selected. The structure and order of the models are selected from the sample using the partial autocorrelation function for the AR part and the autocorrelation function for the MA part of the observed series. However the AR and MA parts of the series are identified when the series is stationary.

For the stationarity test of the data, the unit root and the KPSS test are used and presented in Table 2.

The unit root test tests the null hypothesis that there exist a trend in the series, with a p-value of 0.01694 , the null hypothesis is rejected in favour of the alternative that the data is stationary. On the other hand, the KPSS test, tests the null that the data is stationary and from the p-value of 0.1 , we fail to reject the null hypothesis of stationarity. Thus, the two test results indicate the data is stationary. 
Table 2: Unit root and stationarity test.

\begin{tabular}{cccc}
\hline Test & Statistics & P-value & alpha-level \\
\hline ADF & -3.9277 & 0.01694 & 0.05 \\
KPSS & 0.2642 & 0.1 & 0.05 \\
\hline
\end{tabular}

Series wtt

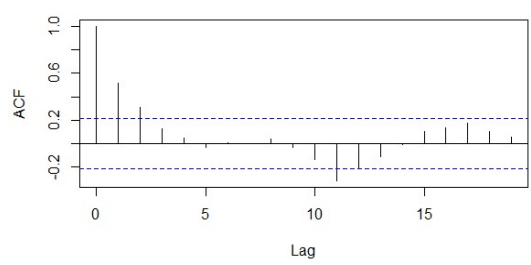

Figure 5: Autocorrelation function. Figure 6: Partial autocorrelation function.

\section{Augmented Dickey-Fuller Test}

Dickey-Fuller $=-3.9277$, Lag order $=4$, p-value $=0.01694$ alternative hypothesis: stationary.

Next we proceed to determine the MA and AR parts from the ACF and PACF respectively presented in Figures 5 and 6.

From Figures 5 and 6 the candid models for the data will be ARIMA $(1,0,1)$ and ARIMA $(1,0,2)$. Since the PACF have spike at lag one and that of the ACF have spikes at 1,2 and 3 .

\section{Model identifications}

In order to identify the appropriate ARIMA models, the values of the AIC's from all the possible models are compared. The best ARIMA model is the one with the minimum AIC value. The ARIMA $(1,0,1)$ recorded an AIC value of 1668.78 in the first experiment. The details of the ARIMA $(1,0,1)$ are presented in Tables 4 and 5. However, the ARIMA $(1,0,2)$ model had a high AIC value of 1670.36 with other details presented in Table 6 . Therefore ARIMA $(1,0,1)$ is the best ARIMA time series model for this data set.

\subsubsection{Performance of model}

In this section we compare the two models and use SSE to choose the best one. The actual monthly solid waste collected by the waste management division of the Kumasi metropolitan Assembly for the year 2015 was obtained. This data set is compared to the 2015 prediction of the Fourier series model and the ARIMA $(1,0,1)$ model. The SSE of both the ARIMA and Fourier series model gives the 
Table 3: Coefficients ARIMA $(1,0,1)$. Table 4: Coefficients ARIMA $(1,0,2)$.

\begin{tabular}{|c|c|c|c|c|c|c|c|c|}
\hline & AR1 & MA1 & MA2 & INTERCEPTS & & AR1 & MA1 & INTERCEPTS \\
\hline & 0.4559 & 0.0298 & 0.1067 & 30094.91 & & 0.5845 & -0.0944 & 30079.651 \\
\hline S.E & 0.2815 & 0.2926 & 0.1589 & 1066.38 & S.E & 0.1553 & 0.1841 & 1112.732 \\
\hline
\end{tabular}

Table 5: Sum of squared error for Fourier series model and the ARIMA model against actual observed.

\begin{tabular}{cccccc}
\hline Month & Fourier series model & ARIMA model & Actual & Fourier S.E. & ARIMA S.E. \\
\hline January & 30066 & 28284 & 24367.7 & 32470167.0 & 15334586.0 \\
February & 28097 & 29030 & 21194.0 & 47650856.8 & 61412112.4 \\
March & 30078 & 29466 & 24584.5 & 30178849.9 & 23830975.3 \\
April & 29039 & 29870 & 22784.8 & 39114642.3 & 50201050.9 \\
May & 26621 & 29870 & 22747.7 & 15002452.8 & 50728439.3 \\
June & 28708 & 29957 & 21462.5 & 52497849.8 & 72160267.8 \\
July & 31576 & 30008 & 21685.5 & 97822781.4 & 69265837.2 \\
August & 30811 & 30038 & 21467.4 & 87303272.0 & 73458476.1 \\
September & 30457 & 30055 & 21142.0 & 86769187.7 & 79445098.6 \\
October & 26608 & 30065 & 25531.2 & 1159485.3 & 2055852.4 \\
November & 22580 & 30071 & 20736.6 & 3398278.16 & 87137408.2 \\
December & 24988 & 30075 & 17906.5 & 50147982 & 148067378.9 \\
SSE & & & & 543515806.04 & 751584183.46 \\
\hline
\end{tabular}

suitability of the two models to the given data. The smaller the SSE the better the model.

The Fourier series produces better forecast of solid waste generation since it has the minimum SSE of 543515806.04 compared with the ARIMA $(1,0,1)$ model which had an SSE of 751584183.46. This optimal performance by the Fourier series model is due to its ability to incorporate the periodicity and the time heterogeneous nature of the solid waste generation data set. This makes our novel approach of forecasting solid waste approach more desirable than existing forecasting approaches.

\subsection{Forecast of solid waste by the Fourier model}

The Fourier series can effectively be used to forecast solid waste generation. We use MATLAB code to implement our proposed model to forecast solid waste generation for the year 2016. The forecasting values are shown in Table 6 . 
Table 6: The expected waste of the year 2016.

\begin{tabular}{cc}
\hline Month & Year 2016 \\
\hline January & 36000 \\
February & 21135 \\
March & 23986 \\
April & 21643 \\
May & 22021 \\
June & 25943 \\
July & 24867 \\
August & 24249 \\
September & 29421 \\
October & 34416 \\
November & 32128 \\
December & 30413 \\
\hline
\end{tabular}

The model gives adequate information on the monthly quantity of solid waste to be expected from January 2016 to December 2016 with a peak value of 36,000 tons occurring in January 2016 and a minimum value of 21,135 tons occurring in February the same year. This informs the decisions of management in putting in adequate measures to address this pertaining issue of waste in the metropolis. Furthermore, the results presented elucidate managers on the human personnel required for the collection, the trucks for solid waste transportation and the size of land required to be used for landfills.

\section{Conclusion}

The Fourier series model has a smaller SSE than that of the ARIMA time series model. This makes Fourier series a more suitable model for forecasting solid waste. Thus, Fourier series has successfully been applied to the problem of forecasting solid waste generation. The periodicity and the time heterogeneous nature of the data set make the Fourier series model suitable for forecasting solid waste generation. Therefore, For data sets that are time heterogeneous and periodic, The Fourier model is more suitable for forecasting.

\section{References}

[1] Amon Mwenda, Dmitry Kuznetsov, Silas Mirau (2014). Time series Forecasting of Solid waste generation in Arusha City-Tanzania.. Vol 4, No 14 (2014) Journal of Mathematical Theory and Modeling, http://www.iiste.org

[2] Benitez S.O., Lozano-Olvera G., Morelos R.A., Vega C.A. (2008). Mathematical modelling to predict residential solid waste generation Waste Manag. 28(1):S7-S13, http://dx.doi.org/10.1016/j.wasman.2008.03.020 
[3] Brian Dyson, Ni-Bin Chang (2005). Forecasting municipal solid waste generation in a fast-growing. Elsevier Waste Management 25 pp 669-690, http://www.ewp.rpi.edu/hartford/

[4] Chen H.W., Ni-Bin Chang (1999). Prediction analysis of Solid waste generation based on Grey fuzzy dynamic modeling.. Elsevier Resources, Conservation and Recycling 29 (2000) pp 1-18.

[5] Ebenezer Owusu-Sekyere, Emmanuel Harris, Ebenezer Bonyah (2013). Forecasting and planning solid waste generation in the Kumasi metropolitan Area of Ghana: An Arima time series approach. International Journal of Sciences Research Article (ISSN 2305-3925) Volume 2, Issue Apr 2013.

[6] Halidi A.L. (2011). Optimization of municipal solid waste management systems: A case of Ilala municipality, Dar es Salaam. University of Dar es Salaam, September, 2011.

[7] Inara Teibe, Ruta Bendere, Larisa Perova, Dace Arina (2002). Mathematical models for Regional Solid Waste Management development. 18th International Conference Linnaeus ECOTECH 2012, At Kalmar, Sweden.

[8] James S. Walker (1991). Fourier Series.

[9] Kumasi metropolitan assembly annual report (2014).

[10] Mohammed B. Oumarou, Mohammed Duada, Abdulbaqi T. Abdulrahim, Alhaji B. Abubakar (2012). Municipal solid waste generation, Recovery and Recycling.. ISSN 2249-0582 World Journal of Engineering and Pure and Applied Sci. 2012; 2(5) pp 143-147.

[11] Nikolaos V. Karadimas, Alessandra Orsoni (2014). Municipal Solid waste Generation Modelling based on fuzzy logic. 20th European conference on modelling and simulation 2006 ISBN 0-95530180-7/ ISBN 0-9553018-15(CD).

[12] Navarro-Esbri, J., Diamadopoulos, E., Ginestar, D. (2002). Time series analysis and forecasting techniques for municipal solid waste management. Greece Resources conservation and recycling. 05/2002; 35(35):201-214. http://dx.doi.org/10.1016/S0921-3449(02)00002-2

[13] Stefano,. A, Marco Franchini, Alberto Marinelli (2007). A shortterm pattern-based model for water-demand forecasting. Journal of Hydroinformatics, Jan 2007, Vol. 9, Issue 1, pp 39-50.

[14] Sarika Rathi (2007). Optimization model for integrated municipal solid waste management in Mumbai, India. Environment and Development Economics, Volume 12, Issue 01, February 2007, pp 105-121, Cambridge University Press, http://dx.doi.org/10.1017/S1355770X0600341X

[15] Tolstov, G.P., Silverman, R.A (2014). A text book on Fourier series.

[16] Trisyanti, D. (2004). Solid waste management of Jakarta, Indonesia. An environmental systems perspective. Diva portal 2004, 84 p , http://www.divaportal.org/smash/get/diva2:411073/FULLTEXT01.pdf

[17] Vatsal Patel, Srinivsarao Meka (2013). Forecasting of Municipal Solid Waste Generation for medium scale towns located in the state of Gujarat, India. International Journal of Innovative Research in Science, Engineering and Technology, Volume 2, Issue 9, September 2013. 